\title{
REVIEW
}

Open Access

\section{Effects of inflammation on the kynurenine pathway in schizophrenia - a systematic review}

\author{
Bruno Pedraz-Petrozzi ${ }^{1,2^{*}}$ (D), Osama Elyamany ${ }^{1,3,6}$, Christoph Rummel ${ }^{4,5}$ and Christoph Mulert ${ }^{1,2,5,6}$
}

\begin{abstract}
Background: In the last decade, there has been growing evidence that an interaction exists between inflammation and the kynurenine pathway in schizophrenia. Additionally, many authors found microglial activation in cases of schizophrenia due to inflammatory mechanisms related mostly to an increase of pro-inflammatory cytokines. In order to gain new insights into the pathophysiology of schizophrenia, it is important to incorporate the latest published evidence concerning inflammatory mechanisms and kynurenine metabolism. This systematic review aims to collect reliable recent findings within the last decade supporting such a theory.
\end{abstract}

Methods: A structured search of electronic databases was conducted for publications between 2008 and 2018 to identify eligible studies investigating patients with schizophrenia/psychosis and the relationship between inflammation and kynurenine pathway. Applicable studies were systematically scored using the NIH Quality Assessment Tools. Two researchers independently extracted data on diagnosis (psychosis/schizophrenia), inflammation, and kynurenine/tryptophan metabolites.

Results: Ten eligible articles were identified where seven studies assessed blood samples and three assessed cerebrospinal fluid in schizophrenic patients.

Of these articles:

- Four investigated the relationship between immunoglobulins and the kynurenine pathway and found correlations between IgA-mediated responses and levels of tryptophan metabolites (i.e., kynurenine pathway).

- Five examined the correlation between cytokines and kynurenine metabolites where three showed a relationship between elevated IL-6, TNF-a concentrations, and the kynurenine pathway.

- Only one study discovered correlations between IL-8 and the kynurenine pathway.

- Two studies showed correlations with lower concentrations of IL-4 and the kynurenine pathway.

- Moreover, this systematic review did not find a significant correlation between CRP ( $n=1$ study), IFN- $\gamma(n=3$ studies), and the kynurenine pathway in schizophrenia.

(Continued on next page)

\footnotetext{
* Correspondence: Bruno.Pedraz@med.uni-giessen.de

${ }^{1}$ Center of Psychiatry, Justus-Liebig University, Klinikstrasse 36, Giessen 35392,

Hessen, Germany

${ }^{2}$ Giessen Graduate School for Life Sciences, Justus-Liebig University,

Leihgesterner Weg 52, Giessen 35392, Hessen, Germany

Full list of author information is available at the end of the article
}

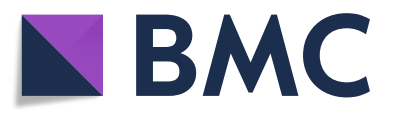

(c) The Author(s). 2020 Open Access This article is distributed under the terms of the Creative Commons Attribution 4.0 International License (http://creativecommons.org/licenses/by/4.0/), which permits unrestricted use, distribution, and reproduction in any medium, provided you give appropriate credit to the original author(s) and the source, provide a link to the Creative Commons license, and indicate if changes were made. The Creative Commons Public Domain Dedication waiver (http://creativecommons.org/publicdomain/zero/1.0/) applies to the data made available in this article, unless otherwise stated. 
(Continued from previous page)

Interpretation: These results emphasize how different inflammatory markers can unbalance the tryptophan/ kynurenine pathway in schizophrenia. Several tryptophan/kynurenine pathway metabolites are produced which can, in turn, underlie different psychotic and cognitive symptoms via neurotransmission modulation. However, due to heterogeneity and the shortage of eligible articles, they do not robustly converge to the same findings. Hence, we recommend further studies with larger sample sizes to elucidate the possible interactions between the various markers, their blood vs. CSF ratios, and their correlation with schizophrenia symptoms.

Keywords: Schizophrenia, Inflammation, Kynurenine, Kynurenic acid, Glutamic acid

\section{Introduction}

Schizophrenia is a chronic disease characterized mostly by psychotic symptoms, cognitive impairment, and functional decline [1]. To date, the pathophysiology of this psychiatric condition remains unclear. However, research on inflammatory factors in schizophrenia has noticeably grown over the last decade [2].

In this context, the vulnerability-stress-inflammation model has been supported by growing evidence $[3,4]$. The model implies that genetic makeup predisposes the subject to be easily affected by stress, which would show inflammatory responses later in life [5]. A review by Lipner et al. has concluded that prenatal maternal stress (e.g., prenatal infection/inflammation, decreased fetal growth, hypoxia-related obstetric complications) has been linked to the development of schizophrenia in offspring [6].

Different animal models have indeed shown correlations with pro-inflammatory cytokines and schizophrenia [7-11]. In addition, authors support immune imbalance in schizophrenia, pointing out a predominance of pro-inflammatory mechanisms [12]. In this case, the presence of different pro-inflammatory metabolites and the inhibition of antiinflammatory factors (e.g., PGJ2) are demonstrated in the onset and chronic phases of schizophrenia [13-15].

From this standpoint, psychotic episodes in schizophrenia coincide with inflammatory mechanisms linked to the hypothalamic-pituitary stress-inflammatory pathways. This eventually leads to microglial and astrocyte activation [16, 17]. Interestingly, the latter is also associated with the activation of the kynurenine pathway and increased the production of kynurenic acid (KYNA) in the cerebrospinal fluid (CSF) $[18,19]$. This metabolite is the only known natural $N$-methyl-D-aspartate (NMDA) receptor antagonist involved in inflammatory processes [20]. This results in the antagonism of the glutamatergic system, which in turn could lead to the dysregulation of dopaminergic neurons. Such changes can be attributed to inflammatory activation $[21,22]$.

In the central nervous system (CNS), the kynurenine pathway starts by the conversion of tryptophan into kynurenine by indoleamine 2,3-dioxygenase 1 (IDO1), IDO2, or tryptophan 2,3-dioxygenase (TDO) (Fig. 1). Astrocytes can express both types of enzymes while microglia express only
IDO $[18,23]$. To a lesser extent, some neurons also possess IDO and/or TDO producing a minor portion of kynurenine [18]. Therefore, kynurenine is available in the CNS via the enzymatic activity of astrocytes, microglia, and some neurons. As well, kynurenine is actively transported into the brain by the large neutral amino acid transporter [19].

Next, kynurenine can follow either of two metabolic branches. First, it can be metabolized into KYNA via kynurenine aminotransferase (KAT) $[19,24,25]$ in astrocytes mainly [26] and neurons through irreversible transamination by KAT [27]. The other branch leads to the formation of quinolinic acid (QUIN) exclusively in both microglia and infiltrating macrophages. Both can express kynurenine 3-monooxygenase (KMO) which is absent in human astrocytes [28]. However, both astrocytes and neurons can further catabolize QUIN, produced by neighboring microglial cells, by the enzyme quinolinate phosphoribosyltransferase (QPRTase) [23]. They can also form the neuroprotective picolinic acid (PIC) as they express the enzyme aminocarboxymuconate semialdehyde decarboxylase (ACMSD) [27].

Considering the previously mentioned discoveries, it is important to integrate inflammatory mechanisms and kynurenine metabolism to gain further insights into the pathophysiology of schizophrenia (i.e., pro-inflammatory cytokines that stimulate the production of KYNA in schizophrenia) [19, 29-31]. In mood disorders, for example, there already exists evidence for correlations between both the kynurenine metabolic pathway and inflammation. One example is bipolar disorder, where microglia are shown to be overactive and levels of KYNA were elevated in the CSF [32-34]. Another example is depression, where patients showed decreased levels of KYNA, QUIN, and kynurenine, correlating with pro-inflammatory cytokines [35-37]. However, in schizophrenia, only a few reviews have focused on inflammation and the kynurenine pathway. One example is the review of Wang and Miller, which considered this correlation but in CSF only [37]. Ribeiro-Santos et al. focused on such a correlation concerning cognitive impairment in schizophrenia [38]. Despite the presence of articles where inflammation and the kynurenine pathway in schizophrenia are reviewed, studies that investigate a broader picture or systematic reviews-including serum and CSF for the 


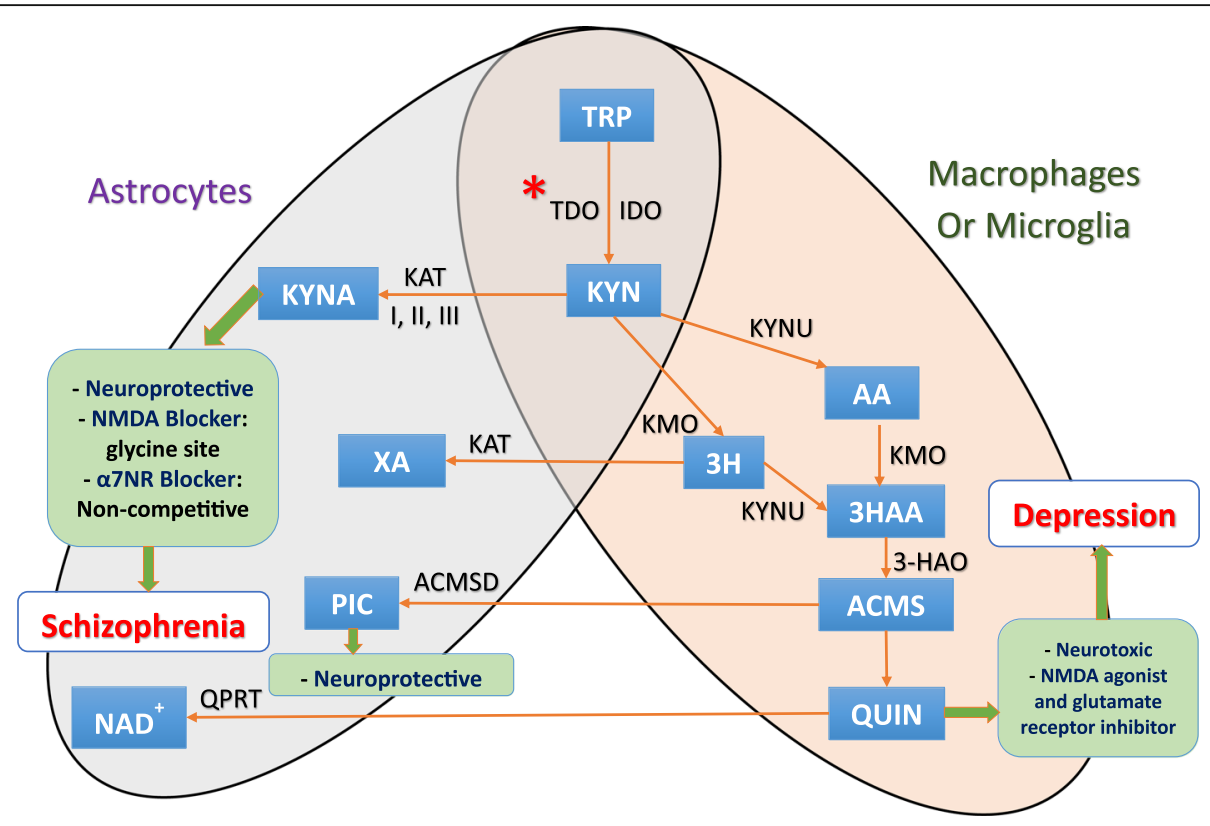

Fig. 1 Kynurenine pathway and tryptophan metabolism in the central nervous system. In the central nervous system (CNS), the kynurenine pathway starts by the conversion of tryptophan into kynurenine by indoleamine 2,3-dioxygenase 1 (IDO1), IDO2, or tryptophan 2,3-dioxygenase (TDO). Astrocytes can express both types of enzymes while microglia express only IDO [18, 23]. To a lesser extent, some neurons also possess IDO and/or TDO producing a minor portion of kynurenine [18]. Therefore, kynurenine is available in the CNS via the enzymatic activity of astrocytes, microglia, and some neurons as well as the kynurenine being actively transported into the brain by the large neutral amino acid transporter [19]. Next, kynurenine can follow either of two metabolic branches. First, it can be metabolized into kynurenic acid (KYNA) via kynurenine aminotransferase (KAT) $[19,24,25]$ in astrocytes mainly [26] and neurons through irreversible transamination by KAT [27]. The other branch leads to the formation of quinolinic acid (QUIN) exclusively in both microglia and infiltrating macrophages. Both can express kynurenine 3-monooxygenase (KMO) which is absent in human astrocytes [28]. However, both astrocytes and neurons can further catabolize QUIN, produced by neighboring microglial cells, by the enzyme quinolinate phosphoribosyltransferase (QPRTase) [23]. They can also form the neuroprotective picolinic acid (PIC) as they express the enzyme aminocarboxymuconate semialdehyde decarboxylase (ACMSD) [27]. Molecules: 3HAA, 3-hydroxyanthranilic; 3HK, 3-hydroxy-kynurenine; AA, anthranilic acid; ACMS, 2-amino-3-carboxymuconate semialdehyde; KYN, kynurenine; KYNA, kynurenic acid; NAD+, nicotinamide adenine dinucleotide; PIC, picolinic acid; QUIN, quinolinic acid; TRP, tryptophan; XA, xanthurenic acid. Enzymes: 3-HAO, 3-hydroxyanthranlic acid oxygenase; ACMSD, aminocarboxymuconate semialdehyde decarboxylase; IDO, indoleamine 2,3dioxygenase; KAT, kynurenine aminotransferase; KMO, kynurenine 3-monooxy-genase; KYNU, kynureninase; QPRT, quinolinic acid phosphoribosyltransferase; TDO, tryptophan-2,3-dioxygenase. *Excitation (examples): tryptophan, T-lymphocytes A4, IFN-a, IFN- $\beta$, IFN- $\gamma$, TNF a. *Inhibition (examples): IL-4, Th2 immunity response, antidepressants, antipsychotics

inflammation markers and the kynurenine pathway-are needed. Indeed, an increasing line of evidence points to such a psycho-neuro-immunology related direction.

To this end, the main aim of this systematic review is to gather state-of-the-art findings in a structured way, based on original research done in the last 10 years on the relationship between inflammation and the kynurenine pathway in schizophrenic patients.

\section{Methods}

\section{Study selection criteria}

Studies of inflammatory cytokines and KYNA in schizophrenia were systematically searched between October and November 2018 using MEDLINE (PubMed, National Center for Biotechnology Information, National Library of Medicine, Maryland, USA), EBSCO (EBSCO Industries Incorporate, Birmingham, AL, USA), Web of Science (Clarivate Analytics, Philadelphia, PA, USA), ProQuest (Cambridge Information Group, Ann Arbor, MI, USA) and
SCIELO (Scientific Electronic Library Online, São Paulo Research Foundation, São Paulo, Brasil), Elsevier ScienceDirect (Elsevier Editorials, Amsterdam, The Netherlands), and Cochrane Library (Cochrane, John Wiley and Sons, Hoboken, NJ, USA).

For the article selection criteria, we only considered original research articles. The search was limited to within the last 10 years (i.e., from 2008 to 2018). The inclusion criteria were as follows: (a) observational studies that assess peripheral blood or CSF inflammatory markers, (b) studies that assess schizophrenia/psychosis and kynurenine pathway metabolites, (c) studies with at least 20 subjects in the patient group with their respective matched control group, (d) studies applied in human species, and (e) studies published in the English language. Studies in human species that included any other kind of methodologies (such as interventional studies, meta-analyses, and reviews of any kind) were excluded. Also, animal experimental studies, studies outside the 
defined time boundaries, studies with too few participants $(<20$ patients), and papers not written in English were excluded.

The risk of bias for each included study was assessed according to the quality assessment tools issued by The National Heart, Lung, and Blood Institute (NHLBI). The selected studies are described and summarized in the "Results" section, as well as presented in tables. The process of selection is highlighted in a PRISMA-Chart (Fig. 2). The sociodemographic data and the study design characteristics for each study included were organized in the tables (Tables 1, 2, and 3). Table 4 shows the application of the NHLBI as quality control for the 10 included studies.

\section{Literature search strategy}

Two authors (BPP and OE) independently conducted a systematic literature search in accordance with the PRISMA (Preferred Reporting Items for Systematic Reviews and Meta-analyses) statement [39].
To select articles that fulfill our main research aim and inclusion criteria, three different categories were introduced. For each of those, similar medical subject headings (MeSH) were included to refine the search, obtaining the following: category 1-schizophrenia ("Psychosis" or "Schizophrenia"), category 2-inflammation ("immune response," "cytokines," "interleukins," or "inflammation"), and category 3-kynurenine ("Kynurenine" or "Kynurenic acid").

Afterwards, the three categories were applied to the search field following this operation: Category 1 (one term) AND Category 2 (one term) AND Category 3 (one term). In this case, we chose one term from each category per each search operation. Hence, the search process resulted in the following Boolean expressions: "Psychosis AND immune response AND Kynurenine"; "Psychosis AND interleukins AND Kynurenine"; "Psychosis AND inflammation AND Kynurenine"; "Psychosis AND cytokines AND Kynurenine"; "Psychosis AND immune response AND Kynurenic acid"; "Psychosis AND interleukins AND Kynurenic acid"; "Psychosis AND inflammation AND Kynurenic acid"; "Psychosis AND cytokines AND
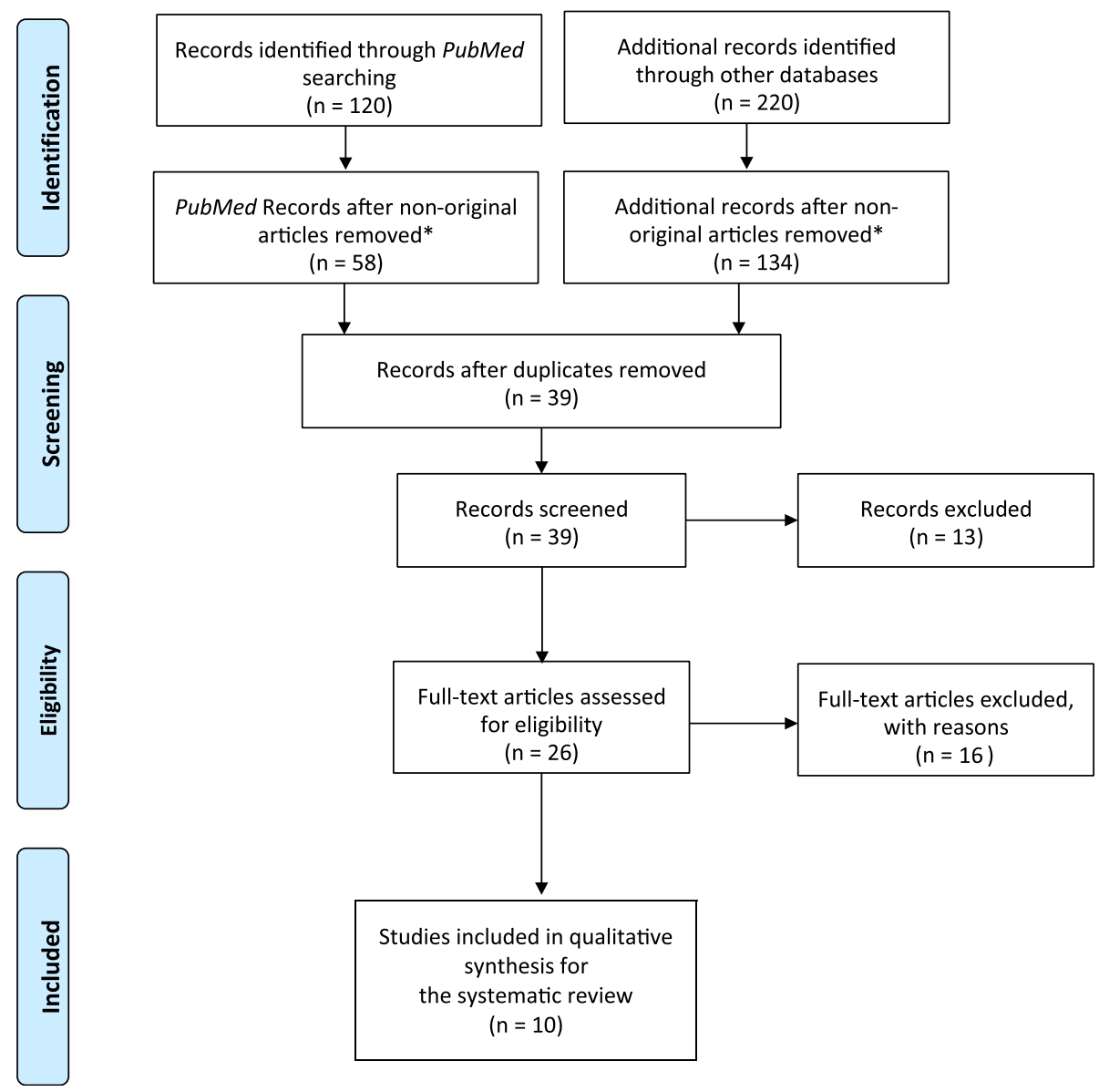

Fig. 2 PRISMA flow diagram. *Non-original articles comprise literature reviews, systematic reviews, meta-analyses, and conference abstracts. From Moher et al. [39] 


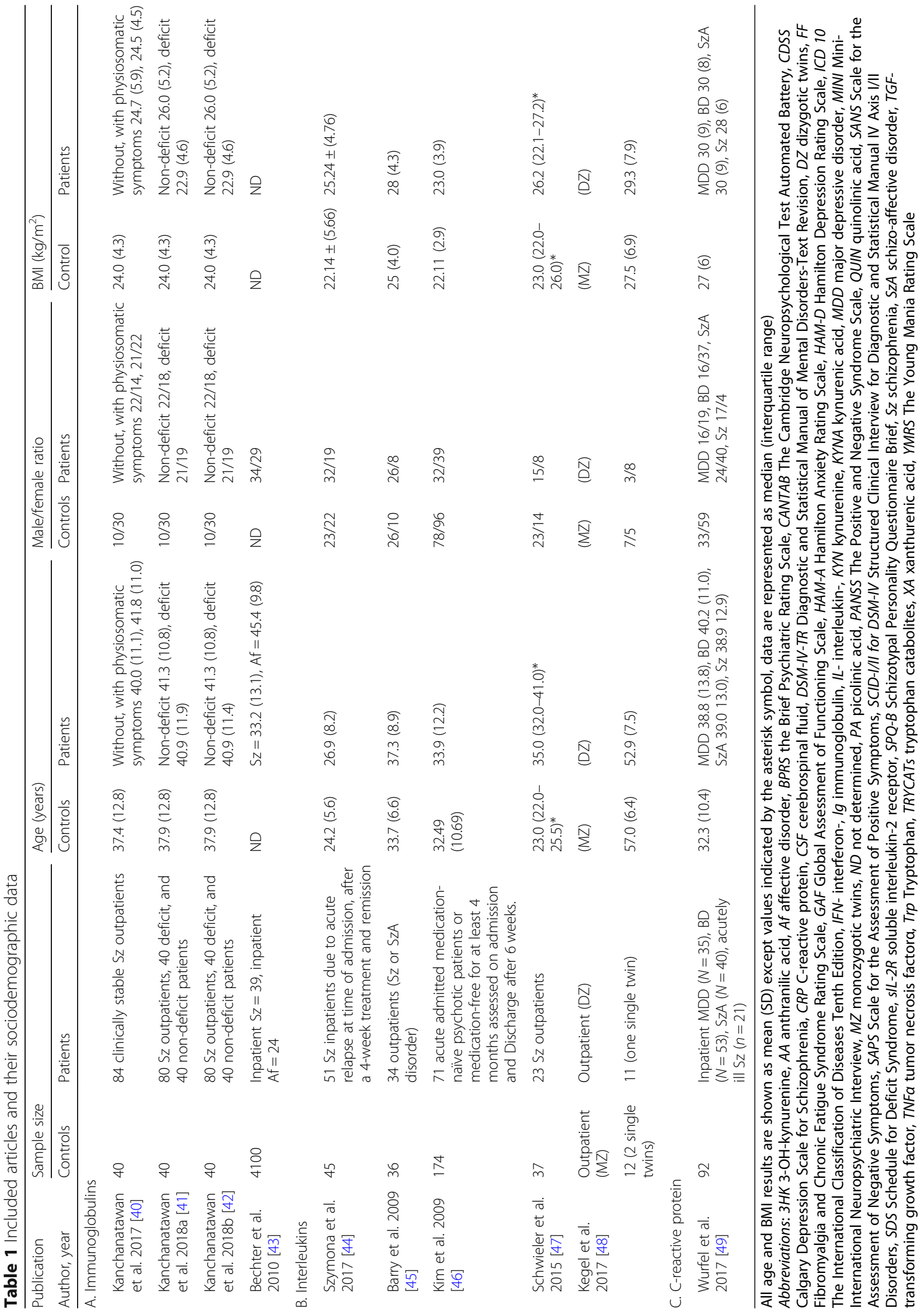




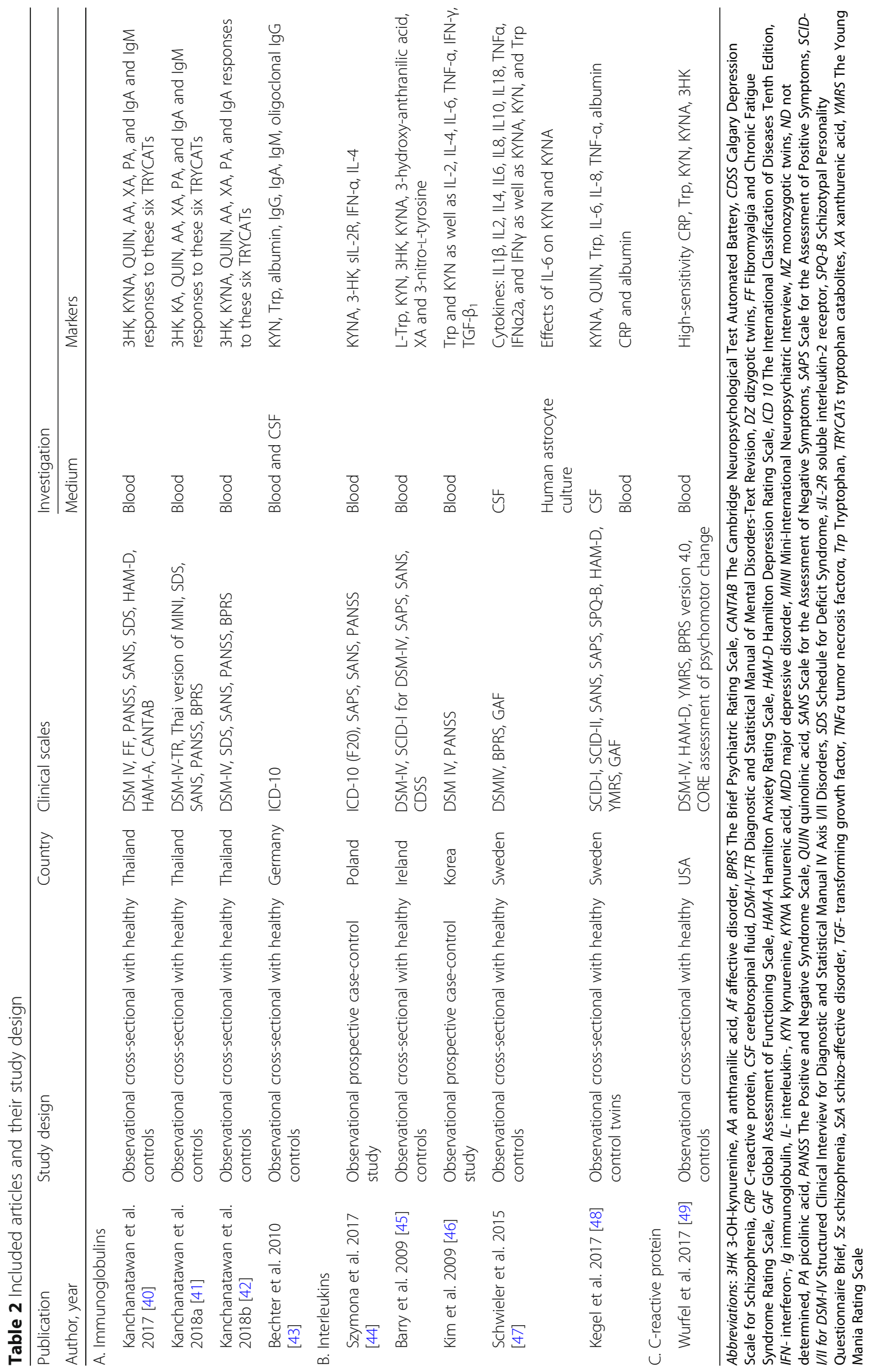




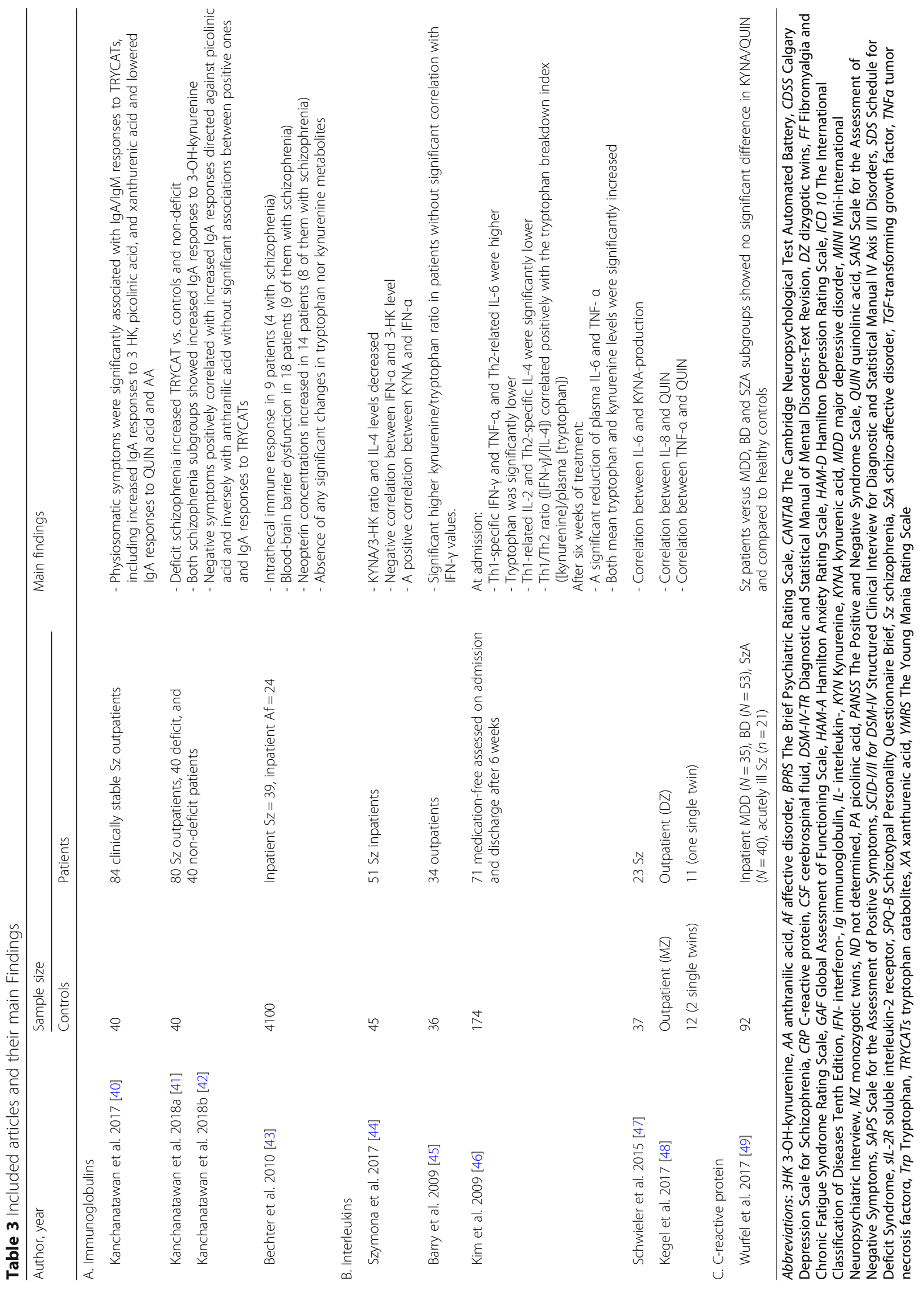


Table 4 Quality assessment for the included studies

\begin{tabular}{|c|c|c|c|c|c|c|c|c|c|c|c|c|c|c|c|}
\hline \multirow[t]{2}{*}{ Year } & \multirow[t]{2}{*}{ Author } & \multicolumn{2}{|l|}{ Sample Size } & \multirow[t]{2}{*}{ Q1 } & \multirow[t]{2}{*}{ Q2 } & \multirow[t]{2}{*}{ Q3 } & \multirow[t]{2}{*}{ Q4 } & \multirow[t]{2}{*}{ Q5 } & \multirow[t]{2}{*}{ Q6 } & \multirow[t]{2}{*}{ Q7 } & \multirow[t]{2}{*}{ Q8 } & \multirow[t]{2}{*}{ Q9 } & \multirow[t]{2}{*}{ Q10 } & \multirow[t]{2}{*}{ Q11 } & \multirow[t]{2}{*}{ Q12 } \\
\hline & & Controls & Patients & & & & & & & & & & & & \\
\hline \multicolumn{16}{|c|}{ A. Immunoglobulins } \\
\hline 2017 & $\begin{array}{l}\text { Kanchanatawan } \\
\text { et al. }\end{array}$ & 40 & 84 clinically stable Sz outpatients & Yes & $C D$ & NR & Yes & Yes & Yes & NR & Yes & NA & Yes & NR & \\
\hline $2018 a$ & $\begin{array}{l}\text { Kanchanatawan } \\
\text { et al. }\end{array}$ & 40 & $\begin{array}{l}80 \text { Sz outpatients, } 40 \text { deficit, and } \\
40 \text { non-deficit patients }\end{array}$ & Yes & Yes & Yes & Yes & Yes & Yes & No & No & Yes & Yes & NA & \\
\hline $2018 b$ & $\begin{array}{l}\text { Kanchanatawan } \\
\text { et al. }\end{array}$ & 40 & $\begin{array}{l}80 \text { Sz outpatients, } 40 \text { deficit, and } \\
40 \text { non-deficit patients }\end{array}$ & Yes & $C D$ & Yes & Yes & Yes & Yes & $N R$ & $C D$ & NA & Yes & Yes & \\
\hline 2010 & Bechter et al. & 4100 & Inpatient $S z=39$, inpatient $A f=24$ & Yes & Yes & No & Yes & Yes & Yes & No & No & Yes & Yes & NA & No \\
\hline \multicolumn{16}{|c|}{ B. Interleukins } \\
\hline 2017 & Szymona et al. & 45 & $\begin{array}{l}51 \text { Sz inpatients due to acute relapse } \\
\text { at time of admission, after a 4-week } \\
\text { treatment and remission }\end{array}$ & Yes & Yes & NR & Yes & Yes & Yes & $N R$ & Yes & NA & Yes & Yes & \\
\hline 2009 & Barry et al. & 36 & 34 outpatients (Sz or SzA disorder & Yes & Yes & No & Yes & Yes & Yes & No & No & Yes & Yes & NA & Yes \\
\hline 2009 & Kim et al. & 174 & $\begin{array}{l}71 \text { acute admitted medication-naïve } \\
\text { psychotic patients or medication-free } \\
\text { for at least } 4 \text { months assessed on } \\
\text { admission and discharge after } 6 \text { weeks. }\end{array}$ & Yes & Yes & NR & Yes & Yes & Yes & NR & $C D$ & NA & Yes & Yes & \\
\hline 2015 & Schwieler et al. & 37 & 23 Sz outpatients & Yes & Yes & No & Yes & Yes & Yes & No & No & Yes & Yes & NA & Yes \\
\hline \multirow[t]{2}{*}{2017} & Kegel et al. & $\begin{array}{l}\text { Outpatient } \\
\text { (MZ) }\end{array}$ & Outpatient (DZ) & Yes & Yes & No & Yes & Yes & Yes & No & No & Yes & Yes & NA & \\
\hline & & $\begin{array}{l}12 \text { ( } 2 \text { single } \\
\text { twins) }\end{array}$ & 11 (one single twin) & & & & & & & & & & & & \\
\hline \multicolumn{16}{|c|}{ C. C-reactive protein } \\
\hline 2017 & Wurfel et al. & 92 & $\begin{array}{l}\text { Inpatient MDD }(N=35), \mathrm{BD}(N=53) \text {, } \\
\text { SzA }(N=40) \text {, acutely ill } S z(n=21)\end{array}$ & Yes & $C D$ & $N R$ & Yes & Yes & Yes & $N R$ & $C D$ & NA & Yes & Yes & \\
\hline
\end{tabular}

Abbreviations: $C D$ cannot determine, $N A$ not applicable, $N R$ not reported, $A f$ affective disorder, $D Z$ dizygotic twins, $M D D$ major depressive disorder, $M Z$ monozygotic twins, Sz schizophrenia, SzA schizo-affective disorder

Questions: Q1. Was the research question or objective in this paper clearly stated? Q2. Was the study population clearly specified and defined? Q3. Was the participation rate of eligible persons at least 50\%? Q4. Were all the subjects selected or recruited from the same or similar populations (including the same time period)? Were inclusion and exclusion criteria for being in the study prespecified and applied uniformly to all participants? Q5. Was a sample size justification, power description, or variance and effect estimates provided? Q6. For the analyses in this paper, were the exposure(s) of interest measured prior to the outcome(s) being measured? Q7. Was the timeframe sufficient so that one could reasonably expect to see an association between exposure and outcome if it existed? Q8. For exposures that can vary in amount or level, did the study examine different levels of the exposure as related to the outcome (e.g., categories of exposure, or exposure measured as continuous variable)? Q9. Were the exposure measures (independent variables) clearly defined, valid, reliable, and implemented consistently across all study participants? Q10. Was the exposure(s) assessed more than once over time? Q11. Were the outcome measures (dependent variables) clearly defined, valid, reliable, and implemented consistently across all study participants? Q12. Were the outcome assessors blinded to the exposure status of participants? Q13. Was loss to follow-up after baseline $20 \%$ or less? Q14. Were key potential confounding variables measured and adjusted statistically for their impact on the relationship between exposure(s) and outcome(s)?

Taken from: The National Heart, Lung and Blood Institute. Study Quality Assessment Tools. Available

at: https://www.nhlbi.nih.gov/health-topics/study-quality-assessment-tools

Kynurenic acid"; "Schizophrenia AND immune response AND Kynurenine"; "Schizophrenia AND interleukins AND Kynurenine"; and "Schizophrenia AND inflammation AND Kynurenine".

\section{Results}

\section{Description of the studies}

Out of 340 candidate publications, our search generated 10 publications, which were included for a qualitative synthesis (Fig. 2). All of the included studies were observational $(n=$ 918). Eight followed a cross-sectional case-control methodology, whereas only two applied a prospective case-control longitudinal method $[44,46]$. The dataset comprised of 918 unique patients from 7 countries (Table 2). The sociodemographic information regarding each study is reported in Table 1 . The characteristics, study design, and main findings for each study are reported in Tables 2 and 3.

Eight studies (80\%) used the Diagnostic and Statistical Manual of Mental Disorders (DSM) criteria to define schizophrenia, and the other two (20\%) considered International Classification of Diseases 10th Version (ICD-10) criteria. Two studies reported additional clinical evaluations by a board-certified psychiatrist involving expert judgment $[48,49]$. 
The patients' exclusion criteria included the following conditions: autoimmune disorders, comorbid psychiatric disorders (mostly substance abuse/dependence), learning disability, use of immunomodulatory non-psychotropic drugs (such as corticosteroids), acute infectious reactions or diseases, allergies, inflammatory response prior to the assessment, leukopenia, neurological disorders (meningoencephalitis, multiple sclerosis, vascular disorders, head injury, epilepsy, Parkinson's disease, Alzheimer's disease, and Huntington's disease), cancer, diabetes mellitus (type 1 and 2), cardiovascular disorder (such as vascular hypertension or past myocardial infarction), inflammatory bowel disease, chronic obstructive pulmonary disease, and finally use of antioxidants and omega $3(\omega 3)$ polyunsaturated fatty acids.

Only 4 out of 10 studies described controls' exclusion criteria precluding the following conditions: personal or family history of psychiatric illness, autoimmune disease, substance abuse/dependence, medical conditions, or concomitant medications likely to influence CNS or immunological function including cardiovascular, respiratory, endocrine, and/or neurological diseases [45, 46, 48, 49]. Only one study disregarded the exclusion of patients with comorbid medical conditions, arguing that their frequency is significantly elevated in a severely ill psychiatric population [49].

Seven out of 10 studies have performed peripheral blood analysis, whereas 3 out of 10 studies have tested CSF. Interferon (IFN-) $\gamma$, IL-2, IL-4, and tumor necrosis factor alpha (TNF- $\alpha$ ) have been measured as inflammatory substances within three publications [44, 46-48]. Other inflammatory substances included IL-6, IL-8, IL-10, IL-18, IFN- $\alpha-2 a$, IFN- $\alpha$, IL- $1 \beta$, C-reactive protein (CRP), immunoglobulin (Ig) M, IgA, and CSF cell counts.

Authors used enzyme-linked immunosorbent assay (ELISA) for the assessment of inflammatory markers. In special conditions when high-sensitive (hs) CRP or some specific markers were measured, authors performed special methodology, for instance, immunoturbidimetry of CRP [49]. High-performance liquid chromatography (HLPC) was the standard method to measure kynurenine and tryptophan metabolites. Variations between studies exclusively depend on technical aspects as well as devices used for analysis.

To assess serum markers, fasting venous blood samples were collected in the morning. Afterwards, the blood samples were centrifuged and stored at $-70{ }^{\circ} \mathrm{C}$. Within the three studies analyzing CSF samples, the authors applied centrifugation, cryopreservation, and ELISA analysis. The intra- and inter-assay coefficients between studies were similar. Detection limits were reported and adequate for respective analyses.

\section{Blood immunoglobulins and kynurenine pathway deregulation in schizophrenia}

Three included studies approached immunoglobulins and the kynurenine pathway deregulation in the serum of schizophrenic patients. For the two of them, information was obtained from one large study divided between two publications. One study classified schizophrenic patients according to the Schedule for the Deficit Syndrome (SDS) into two groups: patients with deficit schizophrenia $(n=40)$ and those with non-deficit schizophrenia $(n=40)$. The authors tried to delineate the differences in tryptophan catabolites' (TRYCATs) profile between both groups in comparison with healthy controls $(n=40)$. Deficit schizophrenia was accompanied by an activated TRYCATs pathway when compared to controls and the non-deficit schizophrenia subgroup [41, 42]. Moreover, both schizophrenia subgroups showed increased IgA responses to 3-OH-kynurenine as compared to controls while KYNA and anthranilic acid (AA) were relatively lowered in the combined schizophrenia groups versus controls with respect to increased levels of noxious TRYCATs $[41,42]$.

The non-deficit schizophrenia patient subgroup versus healthy controls was characterized by relatively increased PIC but lowered QUIN levels [41, 42].

Interestingly, deficit schizophrenia showed much higher IgA responses to PIC, xanthurenic acid (XA), and QUIN; an increased QUIN/KYNA ratio; relatively lowered AA and KYNA levels; and lowered IgA responses to both acids as compared to non-deficit schizophrenia [41, 42].

The negative symptoms of schizophrenia are significantly and positively correlated with increased IgA responses directed against $\mathrm{PIC}$ and inversely with $\mathrm{AA}$, whereas no significant associations between positive symptoms and IgA responses to TRYCATs were found [41, 42].

Another study reported an association between physiosomatic (PS) symptoms and IgA-mediated response in patients with schizophrenia $(n=84)$ in comparison with controls $(n=40)$. PS symptoms, in this case, covers unexplained somatic symptoms (i.e., fatigue, muscle tension, flu-like malaise) related mostly with anxiety and depression. These PS symptoms, which were elevated in more than $50 \%$ of the patients, were significantly associated with IgA/IgM responses to TRYCATs, including increased IgA responses to $3 \mathrm{HK}, \mathrm{PIC}$, and $\mathrm{XA}$ and lowered IgA responses to QUIN and AA [40].

\section{CSF immunoglobulins and kynurenine pathway deregulation in schizophrenia}

Bechter and colleagues [43] analyzed CSF samples from 63 hospitalized patients with schizophrenia $(n=39)$ and affective disorders $(n=24)$ compared with CSF samples from healthy controls $(n=4100)$. They recorded an intrathecal immune response in 9 patients (4 with schizophrenia) in addition to blood-brain barrier dysfunction in 18 patients ( 9 of them with schizophrenia). Neopterin (a human macrophage-produced molecule by IFN- $\gamma$ stimulation) concentrations increased in 14 patients ( 8 of them 
with schizophrenia). However, such augmentation of pro-inflammatory reactions did not lead to significantly detectable changes in tryptophan or kynurenine metabolites [43].

\section{Blood interleukins and kynurenine pathway dysregulation in schizophrenia}

In patients with schizophrenia, the levels of T-helper (Th-) 1-specific IFN- $\gamma$ and TNF- $\alpha$, and Th2-related IL-6 were significantly higher in the plasma of the schizophrenic group $(n=71)$ compared to controls $(n=174)$ while mean plasma tryptophan concentration of the schizophrenic patients was significantly lower in contrast with controls. Moreover, the authors found that Th1related IL-2 and Th2-specific IL-4 were significantly lower in patients' plasma. However, plasma transforming growth factor (TGF-) $\beta 1$ did not show statistically significant difference even though the mean value tended to be higher in schizophrenic patients [46].

In addition, after 6 weeks of treatment with four different atypical antipsychotics (risperidone, amisulpride, olanzapine, and aripiprazole), patients with schizophrenia exhibited a significant reduction of plasma IL-6 and TNF- $\alpha$ [23]. Interestingly, both mean plasma tryptophan and kynurenine levels were significantly increased after 6 weeks of antipsychotic treatment.

Finally, in patients with schizophrenia, the Th1/Th2 ratio (defined as [IFN- $\gamma] /[$ IL-4]) showed a significant positive correlation with admission levels of plasma IL-6 and plasma TNF- $\alpha$. This ratio also positively correlated with the tryptophan breakdown index (defined as plasma [kynurenine]/plasma [tryptophan]) [46].

According to a study conducted in patients with chronic schizophrenia $(n=51)$, compared with healthy control subjects $(n=45)$, the KYNA/3-HK ratio and IL-4 levels, but not soluble IL-2 receptor (sIL-2R) and IFN- $\alpha$ levels, were consistently decreased in schizophrenia patients at all analyzed time points (at time of admission, after a 4-week treatment and remission). Additionally, sIL-2R levels were positively correlated with the number of relapses before treatment [44].

The same study reported that in a subgroup of patients with poor response to pharmacotherapy, initial KYNA levels and the KYNA/3-HK ratio negatively correlated with the number of relapses. Positive association of sIL-2R levels with the number of relapses was also evident in this subgroup; those were treated 4 weeks later with clozapine. Finally, a negative correlation between IFN- $\alpha$ and 3-HK level $(r=-0.875)$ as well as a positive correlation between KYNA and IFN- $\alpha(r=0.472)$ was shown [44].

On the other hand, one out of the 10 studies systematically reviewed here disclosed no statistically significant correlation between kynurenine pathway dysfunction and the presence of interleukins [45]. Despite a significantly higher
KYN/tryptophan ratio in patients compared to healthy controls (patient, $0.055 \pm 0.003$; control, $0.048 \pm 0.002 ; t=$ 2.02 , $\mathrm{df}=68, p<0.05$ ), no statistically significant relationships were revealed between IFN- $\gamma$ values and the KYN/ tryptophan ratio among the groups $(p=0.23)$. The differences of this ratio could not be associated with concomitant IFN- $\gamma$ elevation. Therefore, this study suggests that this increased activity is not IDO mediated [29-31, 45].

\section{CSF interleukins and kynurenine pathway dysregulation in schizophrenia}

The potential role of inflammation and kynurenic acid in psychotic disorders (mostly schizophrenia) was also investigated in twins [48]. Kegel and colleagues discovered a significant association between psychotic symptoms, interleukins, and kynurenine pathway metabolites (measured mostly in CSF) in Swedish-born, same-sex twins discordant for schizophrenia and other psychotic disorders [48]. In detail, they presented a relationship between IL-8 and QUIN (estimate 1.20, standard error [SE] 0.19, $t=6.49$, $p=0.0001$ ) and TNF- $\alpha$ and QUIN (estimate 155, SE 29.7, $t=5.21, p=0.0006)$. By analyzing the intra-pair differences between twins, the authors have revealed associations between kynurenine metabolites and cytokines (QUIN, IL-8, and TNF- $\alpha$ ) in complete twin pairs [48].

Another study by Schwieler and collaborators detected a significant correlation between IL-6 and KYNAproduction in patients with schizophrenia reflected by the tryptophan to KYNA ratio $(r=-0.49 ; p=0.024)$ [47].

Similarly, the authors observed a putative association between elevated IL-6 levels and KYNA in cultured fetal human cortical astrocytes exposed to recombinant human IL-6 $(10 \mathrm{ng} / \mathrm{mL})$. Increased levels of KYNA $48 \mathrm{~h}$ $(1.55 \pm 0.097 \mathrm{nM}$ vs. $1.29 \pm 0.054 \mathrm{nM}, p=0.038)$ and $72 \mathrm{~h}$ $(1.83 \pm 0.070 \mathrm{nM}$ vs. $1.56 \pm 0.053 \mathrm{nM}, p=0.045)$ after IL6 stimulation were detected [47].

\section{Blood CRP and kynurenine pathway dysregulation in schizophrenia}

Only one study exclusively investigated CRP as an inflammatory marker and a possible kynurenine pathway deregulation in acutely ill inpatients with major depressive disorder $(\mathrm{MDD})(N=35)$, bipolar disorder $(\mathrm{BD})$ $(N=53)$, schizoaffective disorder (SZA) $(N=40)$, and schizophrenia $(n=21)$ versus healthy controls $(N=92)$.

Serum KYNA and/or KYNA/QUIN were significantly reduced in all patient subgroups versus healthy controls except for the schizophrenia subgroup, which did not differ from healthy controls [49].

Moreover, a post hoc comparison of patients divided into the categories of non-psychotic affective disorder, affective psychosis, and psychotic disorder (non-affective) manifested reduced levels of KYNA in both the affective disorder and affective psychosis groups. KYNA/3HK was 
significantly reduced in affective psychosis in comparison to non-psychotic affective disorder and in affective psychosis when compared to healthy controls. Similarly, KYNA/ QUIN differed across groups and was significantly reduced in the affective disorder and affective psychosis groups compared with healthy controls. However, no significant difference was shown for patients with schizophrenia.

Follow-up $t$ tests showed that healthy controls had significantly lower CRP concentrations or a statistical trend towards lower CRP concentrations than the MDD, BD, and SZA subgroups. Nonetheless, this was not the case of the patient subgroup with schizophrenia [49].

\section{Discussion}

The results of this study highlight correlations between inflammation, the kynurenine pathway, and schizophrenia in the available literature of human research from the last 10 years. Although few obtained studies related to the specific topic and methodological differences existed between studies, especially concerning sample collection (plasma or CSF), correlations were found between the three mentioned variables.

In regard to studies with only serum samples, correlations between [IFN- $\gamma] /[\mathrm{IL}-4]$ and [kynurenine]/[tryptophan] were found in schizophrenia. Other studies with schizophrenic patients reported correlations between IFN- $\alpha$ and 3-HK levels, as well as correlations between KYNA and IFN- $\alpha$ levels. Additionally, other selected studies found correlations between IgA-mediated responses with levels of tryptophan metabolites (i.e., kynurenine pathway) in schizophrenic patients. Nevertheless, we did not find evidence for a relationship between CRP and tryptophan metabolite levels in patients with schizophrenia.

Regarding studies with only CSF samples, correlations between IL-8 and QUIN, TNF- $\alpha$ and QUIN, and IL-6 and KYNA production were also found in schizophrenia. However, one study demonstrated augmented proinflammatory reactions but without detection of tryptophan metabolites using CSF-cytometry of schizophrenic patients. Figure 1 helps to give a summary of the principal findings on the inflammation effects on the kynurenine pathway in schizophrenia.

\section{Cytokines, kynurenine pathway, and schizophrenia}

Since the discovery of cytokines, our knowledge of signal transmission between cells has been dramatically expanded $[30,50]$. Cytokines have been initially described in systemic inflammatory diseases. Newer evidence suggests that they are also related to other disorders like schizophrenia. The results of the present systematic review revealed the following cytokines as possible candidates for deregulatory mechanisms on the kynurenine pathway in schizophrenia: TNF- $\alpha$, IFN- $\gamma$, IL-6, IL-4, IL8 , and IFN- $\alpha$.

\section{TNF- $a$}

The results showed a correlation between higher TNF- $\alpha$ values and augmented QUIN concentrations in schizophrenia. TNF- $\alpha$ is known for its effect on microglial cells, generating a strong diffuse pro-inflammatory response in the CNS [51]. In addition to that, authors have postulated in the last years that TNF- $\alpha$ modifies the metabolic pathway of tryptophan producing higher quantities of neuroactive substances (i.e., KYNA and QUIN) in the CNS [29-31]. The result of an unbalanced QUIN production could eventually explain cognitive impairment disorders, a clinical entity closely connected to schizophrenia $[4,52,53]$.

\section{IFN- $\gamma$}

The obtained results from this review between IFN- $\gamma$ and kynurenine pathway were ambiguous. It is known that IFN- $\gamma$ activates Th1 cells promoting cytotoxic cell activity and immune responses. Tryptophan catabolism towards KYN formation is mostly mediated by IDO, which is believed to be activated by an increased concentration of IFN- $\gamma$ [54]. In schizophrenia, authors have also postulated an effect of IFN- $\gamma$ on the blockade of NMDA and alpha-7-n-acetylcholinergic receptors through an increase of KYNA concentrations [55, 56]. Moreover, different studies relate IFN- $\gamma$ with an increase of 3 - HK in patients with schizophrenia [57-59]. However, the studies from this systematic review reported two contradictory results. On the one hand, one included study evidenced significant correlations between IFN- $\gamma / \mathrm{IL}-4$ in plasma and tryptophan breakdown ratio ([kynurenine]/ [tryptophan]) in schizophrenia. On the other hand, another included study showed no significant relationships between IFN- $\gamma$ values and the kynurenine/tryptophan ratio. Based on the post-mortem study findings from Miller et al., we suggest that IDO expression does not respond to a concomitant augmentation of IFN- $\gamma$ in the $\mathrm{CNS}$ and that IFN- $\gamma$ eventually has a minor effect on the kynurenine pathway in schizophrenia [60].

\section{IFN- $\boldsymbol{a}$}

The results from this systematic review pointed out a negative correlation between IFN- $\alpha$ and $3-\mathrm{HK}$ but a positive one with KYNA in schizophrenia. Known functions of IFN- $\alpha$ include the stimulation of macrophage antibody-dependent cytotoxicity [51]. Microglia are phagocytic, macrophage-like cells in the CNS and represent the main portion of active innate immune defense in the CNS [61]. Both overactivation and administration of IFN- $\alpha$ induce higher production of KYNA, which can be neurotoxic. Although this effect was published for 
depressive patients, the augmented concentration of IFN- $\alpha$ was shown to unbalance neurophysiological mechanisms also in subjects with schizophrenia [62].

\section{IL-6}

The results of this study found that elevated IL- 6 levels correlated with higher KYNA values in schizophrenia. This cytokine exerts an effect on different proinflammatory mechanisms in microglial cells $[50,51]$ like microglia priming-i.e., the transition from resting to activated state [63]; regulation of the inflammatory response [64]; and overproduction of IL-6 in the aged brain [65]. Due to these microglial functions, authors pointed out that IL-6 was associated with KYNA production by increasing this metabolite in patients with schizophrenia [19]. Other major functions of IL-6 are also the synthesis and promotion of cortisol by hypothalamic stimulation [66]. While such IL-6-action may be related to some other psychiatric disorders like unipolar depression [67], elevation of cortisol production by IL-6-action could also be linked to schizophrenia [68-70]. We suggest that this increased production of cortisol by IL-6-action could be provoked due to two factors: the first one, the exposure to physical or environmental stress, and the second one, genetic vulnerability (i.e., the interaction between these stressors and risk alleles [68]). Both factors, i.e., stress and genetic vulnerability, are components of the mentioned vulnerability-stress model $[4,71,72]$.

\section{IL-8}

The present review shows that this chemokine of the CXC family is related to the kynurenine pathway in schizophrenia. IL-8 contributes to activation and recruitment of neutrophil granulocytes but was also shown to increase QUIN production, as different Alzheimer's disease studies in human post-mortem and serum samples have shown [50,52]. IL-8-induced QUIN induction may be involved in microglial and neuronal activation/damage in the hippocampus eventually contributing to cognitive dysfunction [52]. Although supporting data has been obtained in models designed for Alzheimer's disease research, overproduction of IL-8 and its relation with schizophrenia could also explain cognitive impairment symptoms in patients with schizophrenia [52]. Finally, in vitro studies demonstrated that the combination of TNF- $\alpha /$ IFN- $\gamma$ promotes an augmentation of IL-8 in human astrocytes [73, 74]. Nevertheless, the present review found no significant correlations with IFN- $\gamma$ and positive correlations with TNF- $\alpha$ and kynurenine pathway in patients with schizophrenia.

\section{IL-4}

Only one cytokine from the $\gamma$-chain-cytokine family, namely IL-4, was related to the kynurenine pathway in schizophrenia. It is known that this cytokine is associated with Th2 responses, but it also suppresses KYNA production through blockade of the KAT2 $[50,75]$. The results of the present systematic review highlight that patients with schizophrenia seem to show lower plasma concentration of IL-4. This might lead to an imbalance due to a lower Th2 response and could eventually explain the augmentation of KYNA production in schizophrenia, as previously shown in an animal model for Alzheimer's disease [75].

Interestingly, these results contradict the hypothesis of a predominant $\mathrm{Th} 2$ response in schizophrenia and rather support a shift of the Th cells towards a Th1 response $[72,76]$.

\section{Immunoglobulins, kynurenine pathway, and schizophrenia}

The present review also shows a relationship between IgA-mediated responses and schizophrenia. This antibody plays a crucial role in the immune function of mucous membranes mostly in the gastrointestinal tract providing protection against microbes [77]. Our assessed studies revealed strong correlations between an IgA-mediated response and tryptophan metabolites, especially KYNA and QUIN. Thus, the inflammatory phenomenon most likely unbalances the tryptophan metabolism subsequently contributing to psychotic symptoms.

\section{Negative findings: CRP and CSF-cytometry influence on the kynurenine pathway and schizophrenia}

Our review did not show any significant correlation between CRP and kynurenine metabolism. However, other studies have reported an association between CRP levels and schizophrenia severity [78]. While cytometry of CSF revealed a macrophage-induced pro-inflammatory reaction (i.e., increased neopterin levels) in patients with schizophrenia, a relationship with KYNA or tryptophan metabolites was not found.

\section{Cellular substrates regulating interactions between kynurenine pathway and inflammation \\ Cellular regulation of kynurenine pathway}

Overactivation of astrocytes in schizophrenia was shown to be independent of the patients' medication state [79]. This increased activity of astrocytes in schizophrenia and the persistent reduction of microglial KMO activity result in increased kynurenic acid formation in astrocytes [80]. This finding is consistent with CSF investigation by Schwieler et al. 2015 that showed a positive correlation between IL-6 and KYNA. However, Szymona et al. found decreased KYNA/3-HK ratio in the blood. This could be explained by the KYNA inability to cross the blood-brain barrier.

It is noteworthy that the activation of astrocytes may further facilitate the catabolism of QUIN by QPRTase. 
However, a recent review postulated that in some cases, kynurenine pathway metabolism is likely to be rerouted from KYNA to QUIN production in brain tissue in states of inflammation with microglial activation and macrophage infiltration [81]. Microglial activation was only found in a small percentage of schizophrenia patients and seems to be more pronounced in chronic schizophrenia patients [79]. This might underlie the activation of QUIN pathway in CSF study by Kegel et al. 2017 [48].

\section{Kynurenine pathway and inflammation}

The interaction between intracellular kynurenine pathway and the inflammatory process seems to be bidirectional and more complicated. The first direction is demonstrated by several previous studies showing that inflammatory cytokines can dramatically affect the astrocyte and microglial activity and thus the production of KYNA and QUIN. For example, in vitro studies reveal enhanced IDO-messenger RNA-expression in microglia following IFN- $\gamma$ stimulation, and to a lesser extent in astrocytes [23]. Both IFN- $\gamma$ and TNF- $\alpha$ increase also the production of QUIN by microglia and macrophages [23, 82]. These effects could explain the positive findings found in blood [44-46] and the correlation between TNF- $\alpha$ and QUIN in CSF [48].

In contrast, the opposite direction implies that some kynurenine metabolites can modulate the inflammatory processes. At excitotoxic concentrations, QUIN induces the expression of several pro-inflammatory cytokines and chemokines such as IL-1 $\beta$, monocyte chemotactic protein 1 , and IL-8 in astrocytes [83]. KYNA, an NMDA receptor antagonist, has been shown to act as an immunosuppressant in mice. Such antagonism caused Th1 effector cells to produce less IL-2 and IFN- $\gamma$, whereas it affected Th2 cells to produce more interleukin (IL-) 10 and IL-13. As proposed by a recent review, this interaction suggests a kynurenine pathway-immune feedback loop that may be disrupted in schizophrenia [81]. We suggest that the competition between the pro-inflammatory QUIN (an NMDA receptor agonist produced by microglia) and the immunosuppressant KYNA (an NMDA receptor antagonist) may determine the inflammatory status and/or the clinical picture of certain psychiatric diseases related with neuroinflammation.

\section{Intercellular interactions}

Regarding the intercellular communication, astrocytes produce neuropeptide $\mathrm{Y}$ which inhibits microglial activity. In that sense, astrocytes may inhibit pro-inflammatory cytokine-induced IDO and the microglial kynurenine pathway. In addition, microglial cells mainly secrete type1 cytokines (e.g., IL-12), while astrocytes inhibit the production of pro-inflammatory cytokines and secrete type-2 cytokines (e.g., IL-10).
In the light of our results, in schizophrenic patients, such equilibrium between type 1 and type 2 response in the CNS is disturbed, as showed in the study of Kim et al. [46] and included in this review. It seems that there is an imbalance between the activation of microglial cells and astrocytes [79]. This particular unbalance of the intercellular communication between astrocytes (producing neuroprotective KYNA and PIC) and microglia (producing neurotoxic QUIN) could suggest a competitive interaction between both of them, where the different activation patterns of microglial cells and astrocytes may underlie the biological variations among schizophrenic states.

\section{Limitations and future directions Limitations}

Some limitations have to be taken into account for the present systematic review. First of all, to fulfill our primary aim to find studies assessing the correlation between the "kynurenine pathway," "inflammation," and "schizophrenia," the used Booleans restricted the results; we could not find many studies with other biomarkers, such as enzymes. Nevertheless, this approach enabled us to specifically address the correlations between the three-targeted variables. Second, the studies included in our sample, although they delivered mostly significant results, appeared to be heterogeneous regarding the methodology and patient selection criteria. Third, the definition of schizophrenia varied between studies as it was amenable to the subjectivity of clinical inspection. Independently of the similarities between ICD and DSM classification, standardized protocols to select patients with schizophrenia do not exist and, therefore, cannot guarantee high homogeneity of samples between studies. Finally, some studies did not pay attention to some confounding factors or variables such as duration of illness, doses of antipsychotic drugs, symptom severity, or smoking potentially influencing some of the reviewed results here.

\section{Future directions}

This current article focuses mostly on the influence of inflammation in the tryptophan metabolism in human subjects with schizophrenia. Due to the lack of data on this matter, future studies that investigate the influence of inflammation in the CNS especially related to the kynurenine pathway in schizophrenia are warranted. To integrate different pathways and mechanisms will allow for the possible identification of therapeutic targets and interventions for handling and preventing relapses. While there are differences between studies, the literature overall suggests significant correlations between these three variables.

These findings raise many questions, such as "what are the most important metabolites that relay inflammation 
or inflammatory markers and the kynurenine pathway in schizophrenia?" For example, in an animal model of pregnant rats, the deletion of one allele for KMO $\left(\mathrm{KMO}^{+/-}\right)$in the maternal genetic material brought to a disproportionate KYNA increase in the brain of $\mathrm{KMO}^{+/-}$ offspring rats, compared to $\mathrm{KMO}$ wild-type offspring rats $\left(\mathrm{KMO}^{+/+}\right)$[84]. As mentioned before, in subjects with schizophrenia, the overproduction of KYNA contributes mainly to an imbalance of Th1/Th2 responses. Additionally, in other mouse schizophrenia model, the dysregulation of KYNA explained different cognitive impairments such as anticipatory responses and reduced locomotor activity [85]. The search for understanding this mechanism also in humans, mostly in patients with schizophrenia, will allow to conduct future studies that could regulate the KMO-genetic expression and finally to avoid the imbalance between KNYA and QUIN mechanisms, as showed in patients with schizophrenia.

Additionally, the regulation of NMDA receptors could also have positive effects on schizophrenia, since in the case of schizophrenia KYNA is overexpressed and involved in the receptor antagonism, as well as in the imbalance of inflammatory responses. An experimental model in mice has shown that the regulation of NMDA receptors through KAT2 inhibitors attenuates the KYNA response in the pre-frontal cortex [86]. The prevention of KYNA and the regulation of glutamate release could contribute to the treatment of schizophrenia, mostly to cognitive deficits associated with this mental disorder [86]. Additionally, the regulation of the enzymes related to the kynurenine pathway has suggested to have positive results as an immunomodulatory therapy [87]. Although this data is shown in other disease models, such as depression [87], the stabilization of immune responses in schizophrenia could also contribute to a posterior regulation of noxious effects of the kynurenine pathway, as described before.

What actual therapeutic choices/options could we have in schizophrenia, where a predominant inflammatory factor alters the homeostasis of nerve cells? Currently and to the best of our knowledge, there is no evidence supporting a specific and direct therapeutic option that could regulate the effects of pro-inflammatory cytokines in the kynurenine pathway. What we already know is that some antipsychotics can partially modulate the immune imbalance and the overproduction of KYNA, a natural NMDA receptor antagonist [88]. According to the experimental studies in mice, it is shown that olanzapine regulates the activation of IDO [89]. Nevertheless, the only information we have is related to depression and models for therapeutic targets in schizophrenia regarding this matter are needed. Finally, antiinflammatory drugs (i.e., non-steroid anti-inflammatory drugs) as add-on therapy to antipsychotic therapy have shown regulating effects on psychotic symptomatology, compared to antipsychotic therapy only [88, 90]. However, the detailed pharmacological effects of the add-on anti-inflammatory therapy in schizophrenia remain unknown, and most evidence regarding this topic is inconclusive and, due to the amount of included studies, limited [91]. In this case, more studies are needed to comprehend how does the pharmacology of antiinflammatory drugs work and to understand if these drugs, by inhibiting inflammatory agents, could indirectly or directly influence the kynurenine pathway in schizophrenia.

\section{Conclusions}

In conclusion, the present systematic review found a relationship of elevated IL-6, IL-8, and TNF- $\alpha$ concentrations with the kynurenine pathway in schizophrenia. These higher values could be an explanation for the psychotic symptomatology and cognitive disturbances. However, this systematic review did not find a correlation between CRP, CSF-cytometry, IFN- $\gamma$, and the kynurenine pathway in schizophrenia. With respect to the Th response and lower IL-4 concentrations, we conclude that overactivation of the kynurenine pathway is mostly related to a reduced Th2 and augmented Th1 response. Overall, we conclude that there is a strong immunoglobulin-mediated response against metabolites of the kynurenine pathway, contributing as well to inflammatory mechanisms.

While different methodologies have been applied in the included studies and the results show some heterogeneity, our systematic review gives support to a picture of schizophrenia that integrates inflammatory mechanisms and nerve cell physiology. To expand the understanding of this matter, further studies should be performed.

\footnotetext{
Abbreviations

3HK: 3-OH-kynurenine; AA: Anthranilic acid; ACMSD: Aminocarboxymuconate semialdehyde decarboxylase; Af: Affective disorder; BPRS: The Brief Psychiatric Rating Scale; CANTAB: The Cambridge Neuropsychological Test Automated Battery; CDSS: Calgary Depression Scale for Schizophrenia; CNS: Central nervous system; CRP: C-reactive protein; CSF: Cerebrospinal fluid; DSM-IVTR: Diagnostic and Statistical Manual of Mental Disorders-Text Revision; DZ: Dizygotic twins; FF: Fibromyalgia and Chronic Fatigue Syndrome Rating Scale; GAF: Global Assessment of Functioning Scale; HAM-A: Hamilton Anxiety Rating Scale; HAM-D: Hamilton Depression Rating Scale; ICD 10: The International Classification of Diseases Tenth Edition; IDO: Indoleamine 2,3dioxygenase; IFN-: Interferon-; Ig: Immunoglobulin; IL-: Interleukin-; KAT: Kynurenine aminotransferase; KMO: Kynurenine 3-monooxygenase; KYN: Kynurenine; KYNA: Kynurenic acid; MINI: Mini-International Neuropsychiatric Interview; MDD: Major depressive disorder; MZ: Monozygotic twins; ND: Not determined; NMDA: N-methyl-D-aspartate; PANSS: The Positive and Negative Syndrome Scale; PIC: Picolinic acid; QPRTase: Quinolinate phosphoribosyltransferase; QUIN: Quinolinic acid; SANS: Scale for the Assessment of Negative Symptoms; SAPS: Scale for the Assessment of Positive Symptoms; SCID-I/II for DSM-IV: Structured Clinical Interview for Diagnostic and Statistical Manual IV Axis I/II Disorders; SDS: Schedule for Deficit Syndrome; sIL-2R: Soluble interleukin-2 receptor; SPQ-B: Schizotypal Personality Questionnaire Brief; Sz: Schizophrenia; SzA: Schizo-affective disorder; TDO: Tryptophan 2,3-dioxygenase; TGF: Transforming growth factor; Th: T-helper; TNFa: Tumor necrosis factora;
} 
Trp: Tryptophan; TRYCATs: Tryptophan catabolites; XA: Xanthurenic acid; YMRS: The Young Mania Rating Scale

\section{Acknowledgements}

We would like to thank Jessica Hernandez, a native American Ph.D. student at Justus Liebig University Giessen, for careful English proofreading of the manuscript. This work was funded by the German Research Foundation (Deutsche Forschungsgemeinschaft) - SFB936 (Sonderforschungsbereich 936) - Project C6 - third funding period.

\section{Authors' contributions}

BPP wrote the introduction, methods, results, discussion, and conclusions; corrected the manuscript for the resubmission; and did the literature search. OE corrected the manuscript for the resubmission, did the literature search and prepared the tables. CR corrected the manuscript for the resubmission, proofreading, and paper mentoring. CM:corrected the manuscript for the resubmission, proofreading, and paper mentoring. All authors read and approved the final manuscript.

\section{Authors' information}

BPP: DAAD Member. Third-year doctoral candidate, medical doctor, Justus Liebig University Giessen. Center of Psychiatry, Faculty of Medicine (FB11) Justus-Liebig University in Giessen. Student at Giessen Graduate Life Sciences School (GGL).

OE: SFB936-C6 Member. First-year doctoral candidate, bachelor in medicine and surgery, master in neuroscience, Justus-Liebig University Giessen. Center of Psychiatry, Faculty of Medicine (FB11) - Justus-Liebig University in Giessen Student at Giessen Graduate Life Sciences School (GGL).

CR: apl. Professor at the Institute of Veterinary Physiology and Biochemistry, Faculty of Veterinary Medicine, Justus Liebig University Giessen. Section Speaker, Neurosciences, Giessen Graduate Life Sciences School (GGL). CM: SFB936-C6 Member. Professor and Chairman, Center of Psychiatry, Faculty of Medicine at the Justus-Liebig University in Giessen. Chairman of the Clinic for Psychiatry and Psychotherapy of the University Hospital in Giessen and Marburg (UKGM). Member, Neurosciences, Giessen Graduate Life Sciences School (GGL).

\section{Funding}

This research was performed within the collaborative research center grant SFB936 C6 to C.Mulert and was supported by the German Research Foundation (DFG). Mr. Pedraz-Petrozzi, M.D., is financially supported by the German Exchange Academic Program (DAAD).

\section{Availability of data and materials}

The data that support the findings of this study are available from the corresponding author on request.

\section{Ethics approval and consent to participate} Not applicable

\section{Consent for publication}

Not applicable

\section{Competing interests}

The authors declare that they have no competing interests.

\section{Author details}

${ }^{1}$ Center of Psychiatry, Justus-Liebig University, Klinikstrasse 36, Giessen 35392, Hessen, Germany. ${ }^{2}$ Giessen Graduate School for Life Sciences, Justus-Liebig University, Leihgesterner Weg 52, Giessen 35392, Hessen, Germany. ${ }^{3}$ Alexandria University, 22 El-Guish Road, Alexandria 21526, Alexandria, Egypt. ${ }^{4}$ Institute of Veterinary Physiology and Biochemistry, Justus-Liebig University, Frankfurter Strasse 100, Giessen 35392, Hessen, Germany. ${ }^{5}$ Center for Mind, Brain and Behavior (CMBB), Hans-Meerwein-Strasse 6, Marburg 35043, Hessen, Germany. ${ }^{6}$ Collaborative Research Center 936 (SFB936) - Project C6 Third Funding Period, Justus-Liebig University, Klinikstrasse 36, Giessen 35392 Hessen, Germany.
Received: 7 June 2019 Accepted: 20 January 2020

Published online: 15 February 2020

\section{References}

1. Marder SR, Cannon TD. Schizophrenia. Ropper AH, editor. N Engl J Med. 2019;381(18):1753-61 Available from: http://www.nejm.org/doi/10.1056/ NEJMra1808803. (Cited: 13th November, 2019).

2. Sawa A, Sedlak TW. Oxidative stress and inflammation in schizophrenia. Schizophr Res. 2016;176(1):1-2 Available from: https://inkinghub.elsevier. com/retrieve/pii/S092099641630281X

3. Takahashi T, Nakamura K, Nishiyama S, Furuichi A, Ikeda E, Kido M, et al. Increased pituitary volume in subjects at risk for psychosis and patients with first-episode schizophrenia. Psychiatry Clin Neurosci. 2013;67(7):540-8.

4. Müller N. Inflammation in schizophrenia: pathogenetic aspects and therapeutic considerations. Schizophr Bull. 2018:44(5):973-82 https://doi. org/10.1093/schbul/sby024

5. Müller N. Inflammation in schizophrenia: pathogenetic aspects and therapeutic considerations. Schizophr Bull. 2018;44(5):973-82 Available from: https://academic.oup.com/schizophreniabulletin/article/44/5/973/4965841.

6. Lipner E, Murphy SK, Ellman LM. Prenatal maternal stress and the cascade of risk to schizophrenia spectrum disorders in offspring. Curr Psychiatry Rep. 2019; 21(10):99 Available from: http://link.springer.com/10.1007/s11920-019-1085-1.

7. Meyer U, Feldon J. To poly(l:C) or not to poly(I:C): advancing preclinical schizophrenia research through the use of prenatal immune activation models. Neuropharmacology. 2012;62(3):1308-21 https://doi.org/10.1016/j. neuropharm.2011.01.009.

8. Girgis RR, Kumar SS, Brown AS. The cytokine model of schizophrenia: emerging therapeutic strategies. Biol Psychiatry. 2014;75(4):292-9 Available from: http://linkinghub.elsevier.com/retrieve/pii/S000632231301069X.

9. Smith SEP, Li J, Garbett K, Mirnics K, Patterson PH. Maternal immune activation alters fetal brain development through interleukin-6. J Neurosci. 2007;27(40):10695-702 Available from: http://www.jneurosci.org/cgi/doi/1 0.1523/JNEUROSCI.2178-07.2007.

10. Himmerich H, Sorge S, Kirkby KC, Steinberg H. Schizophrene Störungen: Die Entwicklung immunologischer Krankheits-und Therapiekonzepte. Nervenarzt. 2012;83(1):7-15.

11. Müller N, Schwarz MJ. A psychoneuroimmunological perspective to Emil Kraepelins dichotomy: schizophrenia and major depression as inflammatory CNS disorders. Eur Arch Psychiatry Clin Neurosci. 2008;258(SUPPL. 2):97-106.

12. Martínez-Gras I, Pérez-Nievas BG, García-Bueno B, Madrigal JLM, AndrésEsteban $E$, Rodríguez-Jiménez $R$, et al. The anti-inflammatory prostaglandin $15 \mathrm{~d}-\mathrm{PGJ} 2$ and its nuclear receptor PPARgamma are decreased in schizophrenia. Schizophr Res. 2011;128(1-3):15-22 Available from: https:// linkinghub.elsevier.com/retrieve/pii/S0920996411000521.

13. García-Bueno B, Bioque M, Mac-Dowell KS, Barcones MF, MartínezCengotitabengoa M, Pina-Camacho L, et al. Pro-/anti-inflammatory dysregulation in patients with first episode of psychosis: toward an integrative inflammatory hypothesis of schizophrenia. Schizophr Bull. 2014; 40(2):376-87 Available from: https://academic.oup.com/ schizophreniabulletin/article-lookup/doi/10.1093/schbul/sbt001.

14. Cabrera B, Bioque M, Penadés R, González-Pinto A, Parellada M, Bobes J, et al. Cognition and psychopathology in first-episode psychosis: are they related to inflammation? Psychol Med. 2016;46(10):2133-44.

15. Martinez-Cengotitabengoa M, Macdowell KS, Alberich S, Diaz FJ, GarciaBueno B, Rodriguez-Jimenez $\mathrm{R}$, et al. BDNF and NGF signalling in early phases of psychosis: relationship with inflammation and response to antipsychotics after 1 year. Schizophr Bull. 2016:42(1):142-51.

16. Watanabe Y, Someya T, Nawa H. Cytokine hypothesis of schizophrenia pathogenesis: evidence from human studies and animal models. Psychiatry Clin Neurosci. 2010;64(3):217-30 Available from: http://doi.wiley.com/1 0.1111/j.1440-1819.2010.02094.x.

17. Calcia MA, Bonsall DR, Bloomfield PS, Selvaraj S, Barichello T, Howes OD. Stress and neuroinflammation: a systematic review of the effects of stress on microglia and the implications for mental illness. Psychopharmacology. 2016;233(9):1637-50

18. Müller N, Schwarz MJ. The immunological basis of glutamatergic disturbance in schizophrenia: towards an integrated view. J Neural Transm Suppl. 2007:72:269-80 https://doi.org/10.1007/978-3-211-73574-9_33.

19. Erhardt S, Schwieler L, Imbeault S, Engberg G. The kynurenine pathway in schizophrenia and bipolar disorder. Neuropharmacology. 2017:112:297-306 https://doi.org/10.1016/j.neuropharm.2016.05.020. 
20. Bagasrawala I, Zecevic N, Radonjić NV. N-methyl D-aspartate receptor antagonist kynurenic acid affects human cortical development. Front Neurosci. 2016;30(10):1-15 Available from: http://journalffrontiersin.org/ Article/10.3389/fnins.2016.00435/abstract.

21. Steiner J, Bogerts B, Sarnyai Z, Walter M, Gos T, Bernstein HG, et al. Bridging the gap between the immune and glutamate hypotheses of schizophrenia and major depression: potential role of glial NMDA receptor modulators and impaired bloodbrain barrier integrity. World J Biol Psychiatry. 2012;13(7): 482-92.

22. Plitman E, Iwata Y, Caravaggio F, Nakajima S, Chung JK, Graff-guerrero A. Kynurenic acid in schizophrenia : a systematic review and meta-analysis. Schizophr Bull. 2017;43(4):764-77 https://doi.org/10.1093/schbul/sbw221.

23. Guillemin GJ, Smythe G, Takikawa O, Brew BJ. Expression of indoleamine 2,3-dioxygenase and production of quinolinic acid by human microglia, astrocytes, and neurons. Glia. 2005;49(1):15-23 https://doi.org/10.1002/glia.2 0090.

24. Braidy N, Grant R. Kynurenine pathway metabolism and neuroinflammatory disease. Neural Regen Res. 2017;12(1):39 https://doi.org/10.4103/1673-5374.1 98971.

25. Stavrum AK, Heiland I, Schuster S, Puntervoll P, Ziegler M. Model of tryptophan metabolism, readily scalable using tissue-specific gene expression data. J Biol Chem. 2013;288(48):34555-66 https://doi.org/10.1074/ jbc.M113.474908.

26. Heyes MP, Chen CY, Major EO, Saito K. Different kynurenine pathway enzymes limit quinolinic acid formation by various human cell types. Biochem J. 1997;326(2):351-6 https://doi.org/10.1042/bj3260351.

27. Guillemin GJ, Cullen KM, Lim CK, Smythe GA, Garner B, Kapoor V, et al. Characterization of the kynurenine pathway in human neurons. J Neurosci. 2007;27(47):12884-92 https://doi.org/10.1523/JNEUROSCl.4101-07.2007.

28. Guillemin GJ, Kerr SJ, Smythe GA, Smith DG, Kapoor V, Armati PJ, et al. Kynurenine pathway metabolism in human astrocytes: a paradox for neuronal protection. J Neurochem. 2001;78(4):842-53 https://doi.org/10.1 046/j.1471-4159.2001.00498.x.

29. Dantzer R. Role of the kynurenine metabolism pathway in inflammationinduced depression: preclinical approaches. In: Current topics in behavioral neurosciences; 2016. p. 117-38. Available from: http://link.springer.com/ chapter/10.1007/7854_2011_176.

30. Raison CL, Dantzer R, Kelley KW, Lawson MA, Woolwine BJ, Vogt G, et al. CSF concentrations of brain tryptophan and kynurenines during immune stimulation with IFN-a: relationship to CNS immune responses and depression. Mol Psychiatry. 2010;15(4):393-403 Available from: http://www. nature.com/articles/mp2009116.

31. Arnone D, Saraykar S, Salem H, Teixeira AL, Dantzer R, Selvaraj S. Role of kynurenine pathway and its metabolites in mood disorders: a systematic review and meta-analysis of clinical studies. Neurosci Biobehav Rev. 2018;92: 477-85 https://doi.org/10.1016/j.neubiorev.2018.05.031.

32. Muneer A. Bipolar disorder: role of inflammation and the development of disease biomarkers. Psychiatry Investig. 2016;18(1) Available from: http:// psychiatryinvestigation.org/journal/view.php?doi=10.4306/pi.2016.13.1.18.

33. Fries GR, Walss-Bass C, Bauer ME, Teixeira AL. Revisiting inflammation in bipolar disorder. Pharmacol Biochem Behav. 2019;177:12-9 https://doi.org/1 0.1016/j.pbb.2018.12.006.

34. Olsson S. Elevated levels of kynurenic acid in the cerebrospinal fluid of patients with bipolar disorder. J Psychiatry Neurosci. 2010;35(3):195-9 Available from: http://jpn.ca/jpn/vol35-issue3/195.

35. Ogyu K, Kubo K, Noda Y, Iwata Y, Tsugawa S, Omura Y, et al. Kynurenine pathway in depression: a systematic review and meta-analysis. Neurosc Biobehav Rev. 2018;90:16-25 https://doi.org/10.1016/j.neubiorev.2018.03.023.

36. Raison $\mathrm{CL}$, Miller $\mathrm{AH}$. Role of inflammation in depression: implications for phenomenology, pathophysiology and treatment. In: Inflammation in Psychiatry; 2013

37. Wang AK, Miller BJ. Meta-analysis of cerebrospinal fluid cytokine and tryptophan catabolite alterations in psychiatric patients: comparisons between schizophrenia, bipolar disorder, and depression. Schizophr Bull. 2018:44(1):75-83 Available from: http://academic.oup.com/ schizophreniabulletin/article/44/1/75/3074362.

38. Ribeiro-Santos A, Lucio Teixeira A, Salgado JV. Evidence for an immune role on cognition in schizophrenia: a systematic review. Curr Neuropharmacol. 2014;12(3):273-80.

39. Moher D, Liberati A, Tetzlaff J, Altman DG. Preferred reporting items for systematic reviews and meta-analyses: the PRISMA statement. PLoS Med. 2009;6(7):e1000097 Available from: https://dx.plos.org/10.1371/journal. pmed.1000097. [cited 2019 Nov 10].

40. Kanchanatawan B, Sirivichayakul S, Thika S, Ruxrungtham K, Carvalho A, Geffard M, Anderson G, Noto C, Ivanova R, Maes M. Physio-somatic symptoms in schizophrenia: association with depression, anxiety, neurocognitive deficits and the tryptophan catabolite pathway. Metab Brain Dis. 2017;32(4):1003-16.

41. Kanchanatawan B, Sirivichayakul S, Ruxrungtham K, Carvalho AF, Geffard $M$, Ormstad $H$, et al. Deficit, but not nondeficit, schizophrenia is characterized by mucosa-associated activation of the tryptophan catabolite (TRYCAT) pathway with highly specific increases in IgA responses directed to picolinic, xanthurenic, and quinolinic acid. Mol Neurobiol. 2018;55(2):1524-36 Available from: http://link.springer.com/1 0.1007/s12035-017-0417-6

42. Kanchanatawan B, Sirivichayakul S, Carvalho AF, Anderson G, Galecki P, Maes M. Depressive, anxiety and hypomanic symptoms in schizophrenia may be driven by tryptophan catabolite (TRYCAT) patterning of IgA and IgM responses directed to TRYCATs. Prog Neuro-Psychopharmacol Biol Psychiatry. 2018;80:205-16 Available from: https://linkinghub.elsevier.com/ retrieve/pii/S0278584617301999.

43. Bechter K, Reiber H, Herzog S, Fuchs D, Tumani H, Maxeiner HG. Cerebrospinal fluid analysis in affective and schizophrenic spectrum disorders: identification of subgroups with immune responses and bloodCSF barrier dysfunction. J Psychiatr Res. 2010;44(5):321-30 https://doi.org/1 0.1016/j.jpsychires.2009.08.008.

44. Szymona K, Zdzisińska B, Karakuła-Juchnowicz H, Kocki T, Kandefer-Szerszeń M, Flis M, et al. Correlations of kynurenic acid, 3-hydroxykynurenine, sIL-2R, IFN- $a$, and IL-4 with clinical symptoms during acute relapse of schizophrenia. Neurotox Res. 2017;32(1):17-26 Available from: http://link. springer.com/10.1007/s12640-017-9714-0.

45. Barry S, Clarke G, Scully P, Dinan T. Kynurenine pathway in psychosis: evidence of increased tryptophan degradation. J Psychopharmacol. 2009; 23(3):287-94 Available from: http://journals.sagepub.com/doi/10.1177/026 9881108089583.

46. Kim Y, Myint A, Verkerk R, Scharpe S, Steinbusch H, Leonard B. Cytokine changes and tryptophan metabolites in medication-naïve and medicationfree schizophrenic patients. Neuropsychobiology. 2009;59(2):123-9 Available from: https://www.karger.com/Article/FullText/213565.

47. Schwieler L, Larsson MK, Skogh E, Kegel ME, Orhan F, Abdelmoaty S, et al. Increased levels of IL-6 in the cerebrospinal fluid of patients with chronic schizophrenia - significance for activation of the kynurenine pathway. J Psychiatry Neurosci. 2015;40(2):126-33 Available from: http://jpn.ca/vol40issue2/40-2-126/.

48. Kegel ME, Johansson V, Wetterberg L, Bhat M, Schwieler L, Cannon TD, et al. Kynurenic acid and psychotic symptoms and personality traits in twins with psychiatric morbidity. Psychiatry Res. 2017;247:105-12 Available from: https://linkinghub.elsevier.com/retrieve/pii/S0165178116310472.

49. Wurfel BE, Drevets WC, Bliss SA, McMillin JR, Suzuki H, Ford BN, et al. Serum kynurenic acid is reduced in affective psychosis. Transl Psychiatry. 2017;7(5) https://doi.org/10.1038/tp.2017.88.

50. Akdis M, Burgler S, Crameri R, Eiwegger T, Fujita H, Gomez E, et al. Interleukins, from 1 to 37, and interferon- $\gamma$ : receptors, functions, and roles in diseases. J Allergy Clin Immunol. 2011;127(3):701-721.e70 Available from: https://linkinghub.elsevier.com/retrieve/pii/S0091674910019494.

51. Akdis M, Aab A, Altunbulakli C, Azkur K, Costa RA, Crameri R, et al. Interleukins (from IL-1 to IL-38), interferons, transforming growth factor $\beta$, and TNF-a: receptors, functions, and roles in diseases. J Allergy Clin Immunol. 2016;138(4):984-1010 Available from: https://linkinghub.elsevier. com/retrieve/pii/S0091674916307151.

52. Campbell BM, Charych E, Lee AW, Möller T. Kynurenines in CNS disease: regulation by inflammatory cytokines. Front Neurosci. 2014;8:1-22 Available from: http://journal.frontiersin.org/article/10.3389/fnins.2014.00012/abstract.

53. Müller N, Weidinger $E$, Leitner B, Schwarz MJ. The role of inflammation in schizophrenia. Front Neurosci. 2015;9:372 https://doi.org/10.3389/fnins.2015. 00372.

54. King NJC, Thomas SR. Molecules in focus: indoleamine 2,3-dioxygenase. Int Biochem Cell Biol. 2007;39(12):2167-72 Available from: https://linkinghub. elsevier.com/retrieve/pii/S1357272507000271.

55. Koola MM. Kynurenine pathway and cognitive impairments in schizophrenia: pharmacogenetics of galantamine and memantine. Schizophr Res Cogn. 2016;4:4-9 https://doi.org/10.1016/j.scog.2016.02.001. 
56. Chiappelli J, Notarangelo FM, Pocivavsek A, Thomas MAR, Rowland LM, Schwarcz $R$, et al. Influence of plasma cytokines on kynurenine and kynurenic acid in schizophrenia. Neuropsychopharmacology. 2018:1-6 https://doi.org/10.1038/s41386-018-0038-4

57. Wang $Y, X u$ W, Sun $X$, Chen $Y$, Liu $X$, Tang $H$, et al. Fever of recombinant human interferon-alpha is mediated by opioid domain interaction with opioid receptor inducing prostaglandin E 2. J Neuroimmunol. 2004;156:10712 https://doi.org/10.1016/j.jneuroim.2004.07.013.

58. Johansson A, Owe-Larsson B, Asp L, Kocki T, Adler M, Hetta J, et al. 109. Enhanced kynurenic acid levels and cytokine-induced production of 3hydroxykynurenine in fibroblasts from bipolar or schizophrenic patients. Brain Behav Immun. 2013;32:e31-2 https://doi.org/10.1016/j.bbi.2013.07.121.

59. Grohmann U, Fallarino F, Puccetti P. Tolerance, DCs and tryptophan: Much ado about IDO. Trends Immunol. 2003;24:242-8 Available from: http:// linkinghub.elsevier.com/retrieve/pii/S1471490603000723.

60. Miller CL, Llenos IC, Dulay JR, Barillo MM, Yolken RH, Weis S. Expression of the kynurenine pathway enzyme tryptophan 2,3-dioxygenase is increased in the frontal cortex of individuals with schizophrenia. Neurobiol Dis. 2004; 15(3):618-29 Available from: https://linkinghub.elsevier.com/retrieve/pii/S096 9996104000026

61. Filiano AJ, Gadani SP, Kipnis J. Interactions of innate and adaptive immunity in brain development and function. Brain Res. 2015;1617:18-27 https://doi. org/10.1016/j.brainres.2014.07.050.

62. Wichers MC, Koek GH, Robaeys G, Verkerk R, Scharpé S, Maes M. IDO and interferon-a-induced depressive symptoms: a shift in hypothesis from tryptophan depletion to neurotoxicity. Mol Psychiatry. 2005;10(6):538-44 Available from: http://www.nature.com/articles/4001600.

63. Perry $\mathrm{VH}$, Holmes C. Microglial priming in neurodegenerative disease. Nat Rev Neurol. 2014;10(4):217-24 https://doi.org/10.1038/nrneurol.2014.38.

64. Nakazato R, Hotta S, Yamada D, Kou M, Nakamura S, Takahata Y, et al. The intrinsic microglial clock system regulates interleukin-6 expression. Glia. 2017;65(1):198-208 Available from: http://doi.wiley.com/10.1002/glia.23087.

65. Ye S-M, Johnson RW. Increased interleukin-6 expression by microglia from brain of aged mice. J Neuroimmunol. 1999;93(1-2):139-48 Available from: https://linkinghub.elsevier.com/retrieve/pii/S0165572898002173.

66. Gadek-Michalska A, Bugajski J. Interleukin-1 (IL-1) in stress-induced activation of limbic-hypothalamic-pituitary adrenal axis. Pharmacol Rep. 2010;62(6): 969-82 Available from: https://linkinghub.elsevier.com/retrieve/pii/S1734114 010703595

67. Köhler CA, Freitas TH, Maes M, de Andrade NQ, Liu CS, Fernandes BS, et al. Peripheral cytokine and chemokine alterations in depression: a metaanalysis of 82 studies. Acta Psychiatr Scand. 2017;135(5):373-87.

68. Chiappelli J, Shi Q, Kodi P, Savransky A, Kochunov P, Rowland LM, et al. Disrupted glucocorticoid-immune interactions during stress response in schizophrenia. Psychoneuroendocrinology. 2016;63:86-93 Available from: https://linkinghub.elsevier.com/retrieve/pii/S0306453015009130.

69. Simpson RJ, Hammacher A, Smith DK, Matthews JM, Ward LD. Interleukin-6: structure-function relationships. Protein Sci. 1997;6(5):929-55 Available from: http://doi.wiley.com/10.1002/pro.5560060501.

70. Anderson G, Kubera M, Duda W, Lasoń W, Berk M, Maes M. Increased IL-6 trans-signaling in depression: focus on the tryptophan catabolite pathway, melatonin and neuroprogression. Pharmacol Rep. 2013;65(6):1647-54 Available from: http://www.ncbi.nlm.nih.gov/pubmed/24553013.

71. Brydon L, Walker C, Wawrzyniak A, Whitehead D, Okamura H, Yajima J, et al. Synergistic effects of psychological and immune stressors on inflammatory cytokine and sickness responses in humans. Brain Behav Immun. 2009;23(2): 217-24 https://doi.org/10.1016/j.bbi.2008.09.007.

72. Müller N, Schwarz MJ. Immunologische aspekte bei schizophrenen störungen. Nervenarzt. 2007;78(3):253-63.

73. Croitoru-Lamoury J, Guillemin GJ, Boussin FD, Mognetti B, Gigout LI, Chéret $A$, et al. Expression of chemokines and their receptors in human and simian astrocytes: evidence for a central role of TNFa and IFNy in CXCR4 and CCR5 modulation. Glia. 2003;41(4):354-70 Available from: http://doi.wiley.com/10.1 002/glia.10181.

74. Guillemin GJ, Croitoru-Lamoury J, Dormont D, Armati PJ, Brew BJ. Quinolinic acid upregulates chemokine production and chemokine receptor expression in astrocytes. Glia. 2003;41(4):371-81 Available from: http://doi. wiley.com/10.1002/glia.10175.

75. Papadimitriou C, Celikkaya H, Cosacak MI, Mashkaryan V, Bray L, Bhattarai P, et al. 3D culture method for Alzheimer's disease modeling reveals
Interleukin-4 rescues Aß42-induced loss of human neural stem cell plasticity. Dev Cell. 2018;46(1):85-101.e8 https://doi.org/10.1016/j.devcel.2018.06.005.

76. Schwarz MJ, Müller N, Riedel M, Ackenheil M. The Th2-hypothesis of schizophrenia: a strategy to identify a subgroup of schizophrenia caused by immune mechanisms. Med Hypotheses. 2001;56(4):483-6.

77. Fagarasan S, Honjo T. Intestinal IgA synthesis: regulation of front-line body defences. Nat Rev Immunol. 2003;3(1):63-72 Available from: http://www. nature.com/articles/nri982.

78. Faugere M, Micoulaud-Franchi J-A, Faget-Agius C, Lançon C, Cermolacce M, Richieri R. High C-reactive protein levels are associated with depressive symptoms in schizophrenia. J Affect Disord. 2018;225:671-5 https://doi.org/1 0.1016/j.jad.2017.09.004.

79. Müller N, Myint AM, Krause D, Weidinger E, Schwarz MJ. Anti-inflammatory treatment in schizophrenia. Prog Neuro-Psychopharmacology Biol Psychiatry. 2013;42:146-53 https://doi.org/10.1016/j.pnpbp.2012.11.008.

80. Wonodi I, Schwarcz R. Cortical kynurenine pathway metabolism: a novel target for cognitive enhancement in schizophrenia. Schizophr Bull. 2010; 36(2):211-8 https://doi.org/10.1093/schbul/sbq002.

81. de Bie J, Lim CK, Guillemin GJ. Kynurenines, gender and neuroinflammation; showcase schizophrenia. Neurotox Res. 2016;30(3):285-94 https://doi.org/1 0.1007/s12640-016-9641-5.

82. Pemberton LA, Kerr SJ, Smythe G, Brew BJ. Quinolinic acid production by macrophages stimulated with IFN-gamma, TNF-alpha, and IFN-alpha. J Interf Cytokine Res. 1997;17(10):589-95 https://doi.org/10.1089/jir.1997.17.589.

83. Guillemin GJ. Quinolinic acid, the inescapable neurotoxin. FEBS J. 2012; 279(8):1356-65 https://doi.org/10.1111/j.1742-4658.2012.08485.x

84. Beggiato S, Notarangelo FM, Sathyasaikumar KV, Giorgini F, Schwarcz R. Maternal genotype determines kynurenic acid levels in the fetal brain: implications for the pathophysiology of schizophrenia. J Psychopharmacol. 2018;32(11):1223-32 https://doi.org/10.1177/0269881118805492.

85. Hahn B, Reneski CH, Pocivavsek A, Schwarcz R. Prenatal kynurenine treatment in rats causes schizophrenia-like broad monitoring deficits in adulthood. Psychopharmacology. 2018;235(3):651-61 https://doi.org/10.1 007/s00213-017-4780-9.

86. Bortz DM, Wu HQ, Schwarcz R, Bruno JP. Oral administration of a specific kynurenic acid synthesis (KAT II) inhibitor attenuates evoked glutamate release in rat prefrontal cortex. Neuropharmacology. 2017;121:69-78 https:// doi.org/10.1016/j.neuropharm.2017.04.023.

87. Molteni R, Macchi F, Zecchillo C, Dell'Agli M, Colombo E, Calabrese F, et al. Modulation of the inflammatory response in rats chronically treated with the antidepressant agomelatine. Eur Neuropsychopharmacol. 2013;23(11): 1645-55 https://doi.org/10.1016/j.euroneuro.2013.03.008.

88. Muller N, Myint A-M, J. Schwarz M. Kynurenine pathway in schizophrenia: pathophysiological and therapeutic aspects. Curr Pharm Des. 2011;17(2): 130-6 https://doi.org/10.2174/138161211795049552.

89. Thomas J, Khanam R, Vohora D. Activation of indoleamine 2, 3- dioxygenase pathway by olanzapine augments antidepressant effects of venlafaxine in mice. Psychiatry Res. 2017:258:444-8 https://doi.org/10.1016/j.psychres.2017. 08.083.

90. Zheng W, Cai D, Bin YXH, Ungvari GS, Ng CH, Müller N, et al. Adjunctive celecoxib for schizophrenia: a meta-analysis of randomized, double-blind, placebo-controlled trials. J Psychiatr Res. 2017;92:139-46 https://doi.org/10.1 016/j.jpsychires.2017.04.004.

91. Schmidt L, Phelps E, Friedel J, Shokraneh F. Acetylsalicylic acid (aspirin) for schizophrenia. Cochrane Database Syst Rev. 2019;2016:10(3) Available from: http://doi.wiley.com/10.1002/14651858.CD012116.

\section{Publisher's Note}

Springer Nature remains neutral with regard to jurisdictional claims in published maps and institutional affiliations. 